\title{
HOMOGENIZATION OF TRANSPORT PROCESSES AND HYDRATION PHENOMENA IN FRESH CONCRETE
}

\author{
Michal Beneš ${ }^{a}$, RADEK ŠTeFAN ${ }^{b, *}$ \\ ${ }^{a}$ Czech Technical University in Prague, Faculty of Civil Engineering, Department of Mathematics, Thákurova 7, \\ 16629 Prague 6, Czech Republic \\ ${ }^{b}$ Czech Technical University in Prague, Faculty of Civil Engineering, Department of Concrete and Masonry \\ Structures, Thákurova 7, 16629 Prague 6, Czech Republic \\ * corresponding author: radek.stefan@fsv.cvut.cz
}

ABstract.

The problem of hydration and transport processes in fresh concrete is strongly coupled and non-linear, and therefore, very difficult for a numerical modelling. Physically accurate results can be obtained using fine-scale simulations, which are, however, extremely time consuming. Therefore, there is an interest in developing new physically accurate and computationally effective models. In this paper, a new fully coupled two-scale (meso-macro) homogenization framework for modelling of simultaneous heat transfer, moisture flows, and hydration phenomena in fresh concrete is proposed. A modified mesoscale model is first introduced. In this model, concrete is assumed as a composite material with two periodically distributed mesoscale components, cement paste and aggregates. A homogenized model is then derived by an upscaling method from the mesoscale model. The coefficients for the homogenized model are obtained from the solution of a periodic cell problem. For solving the periodic cell problem, two approaches are used - a standard finite element method and a simplified closed-form approximation taken from literature. The homogenization framework is then implemented in MATLAB environment and finally employed for illustrative numerical experiments, which verify that the homogenized model provides physically accurate results comparable with the results obtained by the mesoscale model. Moreover, it is verified that, using the homogenization framework with a closed-form approach to the periodic cell problem, significant computational cost savings can be achieved.

KEYWORDS: Concrete, hydration, transport processes, homogenization, numerical experiments.

\section{INTRODUCTION}

Modelling of transport processes and hydration phenomena in early age concrete is a challenging engineering problem, since hydration of cement is an exothermic reaction, which may result in high temperatures and related deformations, stresses, and cracking in concrete structures, e.g. [1/7] and references therein.

Fresh concrete is a heterogeneous porous material highly saturated with liquid water, e.g. 44. In the present paper, concrete is assumed as a composite material with two periodically distributed mesoscale components, cement paste and aggregates, represented by multiphase hygroscopic and heat conducting rigid porous media partially saturated with liquid water, e.g. [4, 8].

Coupled transport processes in porous materials are associated with balance equations for mass of moisture and heat energy of the whole medium, e.g. [9]. For the mesoscopic description of transport processes in cement paste and aggregates, we use the balance equations based on the averaging theory, see 4 and references therein, particularly [10 12], for more details. The governing equations at the mesoscale level are completed by an appropriate set of constitutive equa- tions, material data, and physically relevant boundary and initial conditions.

It is well known and closely described in literature that the problem of hydration and transport processes in concrete is strongly coupled and non-linear, e.g. [4, 7] and references therein. This, together with the complexity of the mesoscopic structure of the concrete composite, makes the detailed numerical simulations of the problem extremely time consuming. Therefore, there is an interest in developing new physically accurate and computationally effective models based on a sophisticated numerical coupling at different scales, see e.g. [13 16], see also our previous work [17, 18].

The homogenization method is one of the most advanced techniques in upscaling the response of the microstructure of heterogeneous materials, e.g. [1928. In this method, the solution of a fine scale problem is used to examine the local material behaviour at the macroscale. Combining the heat transfer together with mass flows through fresh concrete motivates us to construct a fully coupled multi-field and a two-scale (meso-macro) homogenization framework. 


\section{The Mesoscale MODEL}

In the mesoscale model, concrete is assumed as a composite material with two periodically distributed mesoscale components, cement paste and aggregates, represented by multiphase hygroscopic and heat conducting rigid porous media partially saturated with liquid water, e.g. 44, 8. The detailed description of the assumptions adopted for developing the model is given in our previous work [17].

Let $\Omega$ be a polygonal domain with boundary $\partial \Omega$. Consider a concrete composite consisting of two flow regions (aggregates and cement paste) periodically distributed in a domain $\Omega$ with period $\varepsilon \mathcal{Y}$, where $\mathcal{Y}=(0,1)^{2}$ is a periodicity cell split into two complementary parts $\mathcal{Y}_{a}$ and $\mathcal{Y}_{c}$. Throughout the paper, subscripts $a$ and $c$ refer to aggregates and cement paste, respectively. By $\chi_{a}$ and $\chi_{c}$, we denote the corresponding characteristic functions of $\mathcal{Y}_{a}$ and $\mathcal{Y}_{c}$, respectively, extended $\mathcal{Y}$-periodically to $\mathbb{R}^{2}$. From the geometrical point of view, $\varepsilon$ is the characteristic length representing the small scale variability of the concrete composite. In this paper, we present the homogenization result for the problem (indexed by $\varepsilon$ )

$$
\begin{aligned}
& \partial_{t}\left[\varrho_{w}\left(\chi_{c}^{\varepsilon} \phi_{c}\left(\xi^{\varepsilon}\right)+\chi_{a}^{\varepsilon} \phi_{a}\right) \mathcal{S}\left(p^{\varepsilon}\right)\right] \\
& -\nabla \cdot\left[\frac{\varrho_{w} k_{R}\left(\mathcal{S}\left(p^{\varepsilon}\right)\right)}{\mu\left(\vartheta^{\varepsilon}\right)}\left(\chi_{c}^{\varepsilon} k_{c}\left(\xi^{\varepsilon}\right)+\chi_{a}^{\varepsilon} k_{a}\right) \nabla p^{\varepsilon}\right] \\
= & \alpha_{1} \chi_{c}^{\varepsilon} f\left(p^{\varepsilon}, \vartheta^{\varepsilon}, \xi^{\varepsilon}\right),
\end{aligned}
$$

$$
\begin{aligned}
& \partial_{t}\left[c_{w} \varrho_{w}\left(\chi_{c}^{\varepsilon} \phi_{c}\left(\xi^{\varepsilon}\right)+\chi_{a}^{\varepsilon} \phi_{a}\right) \mathcal{S}\left(p^{\varepsilon}\right) \vartheta^{\varepsilon}\right] \\
& +\partial_{t}\left[\left(\chi_{c}^{\varepsilon} \varrho_{s c} c_{s c}\left(1-\phi_{c}\left(\xi^{\varepsilon}\right)\right)+\chi_{a}^{\varepsilon} \varrho_{s a} c_{s a}\left(1-\phi_{a}\right)\right) \vartheta^{\varepsilon}\right] \\
& -\nabla \cdot\left[\left(\chi_{c}^{\varepsilon} \lambda_{c}\left(p^{\varepsilon}, \vartheta^{\varepsilon}, \xi^{\varepsilon}\right)+\chi_{a}^{\varepsilon} \lambda_{a}\left(p^{\varepsilon}, \vartheta^{\varepsilon}\right)\right) \nabla \vartheta^{\varepsilon}\right] \\
& -\nabla \cdot\left[c_{w} \varrho_{w} \vartheta^{\varepsilon} \frac{k_{R}\left(\mathcal{S}\left(p^{\varepsilon}\right)\right)}{\mu\left(\vartheta^{\varepsilon}\right)}\left(\chi_{c}^{\varepsilon} k_{c}\left(\xi^{\varepsilon}\right)+\chi_{a}^{\varepsilon} k_{a}\right) \nabla p^{\varepsilon}\right] \\
& =\alpha_{2} \chi_{c}^{\varepsilon} f\left(p^{\varepsilon}, \vartheta^{\varepsilon}, \xi^{\varepsilon}\right)
\end{aligned}
$$

coupled with an integral condition

$$
\xi^{\varepsilon}(x, t)=\int_{0}^{t} f\left(p^{\varepsilon}(x, s), \vartheta^{\varepsilon}(x, s), \xi^{\varepsilon}(x, s)\right) \mathrm{d} s .
$$

The unknowns in the model are the temperature $\vartheta^{\varepsilon}$, water pressure $p^{\varepsilon}$, and the memory function $\xi^{\varepsilon}$, the so-called degree of hydration. From the physical point of view, equations (1) and (2), respectively, represent the mass balance of moisture (liquid water) and the heat equation for the porous system, see e.g. 9]. Equation (3) represents an additional integral condition. Such type of equations arises in the theory of heat conduction when inner heat sources are of special types, in particular, in so-called problems of hydration heat. In this case, the intensity of inner sources of heat also depends on the amount of heat already developed, see 29]. In the mesoscale model, we assume different hydraulic and thermal characteristics in aggregate and cement paste, respectively. In particular, $\phi_{\square}(\square \approx a, c)$ is the porosity, and $\mathcal{S}$ represents the degree of saturation with liquid water. Further, $k_{\square}$ is the intrinsic permeability, $k_{R}$ represents the relative hydraulic conductivity, $\lambda_{\square}$ is the thermal conductivity function, and $\mu$ is the temperature dependent viscosity of the fluid. Material constants are as follows: $\varrho_{w}$ is the density of liquid water and $c_{w}$ represents the isobaric heat capacity of water. Moreover, $\varrho_{s} \square$ and $c_{s}$, respectively, are the mass density and the isobaric heat capacity of the solid. Finally, $f$ is the hydration degree rate and parameters $\alpha_{1}$ and $\alpha_{2}$ on the right hand sides of (1) and (2) express the final values of mass of hydrated water and the amount of released heat energy during hydration, respectively.

To complete the model, it is further necessary to prescribe the initial and boundary conditions. The initial conditions specify the initial fields of the water pressure and temperature:

$$
p^{\varepsilon}(0)=p_{0}, \quad \vartheta^{\varepsilon}(0)=\vartheta_{0} \quad \text { in } \Omega .
$$

The so-called Newton (Robin type) boundary conditions are prescribed on the boundary $\partial \Omega$ where the water flux through the boundary of the domain is proportional to the difference between the pressure at the boundary and the outer pressure $p_{\infty}$. Similarly, the heat flux at the boundary of the system is proportional to the difference between the temperature at the boundary and the external temperature $\vartheta_{\infty}$.

\section{THE HOMOGENIZED PROBLEM}

The system (1) - (3) has been theoretically investigated in [17, 29]. Instead of solving (1)-(3) on a fine mesh resolving the small scale variability of $\varepsilon$, the basic idea of the upscaling methods is to replace the heterogeneous medium by an "equivalent" homogeneous one. It has been shown in 17] that there is a sequence $\left\{\varepsilon_{j}\right\}$, $\lim _{j \rightarrow+\infty} \varepsilon_{j}=0^{+}$, such that $\left[p^{\varepsilon_{j}}, \vartheta^{\varepsilon_{j}}, \xi^{\varepsilon_{j}}\right]$ converges in a suitable topology to the solution of the following upscaled equations.

Water conservation equation:

$$
\begin{aligned}
\partial_{t}\left[\varrho_{w}\left(\chi_{c}^{*} \phi_{c}(\xi)+\chi_{a}^{*} \phi_{a}\right) \mathcal{S}(p)\right] \\
-\nabla \cdot\left[\boldsymbol{A}^{*}(p, \vartheta, \xi) \nabla p\right]=\alpha_{1} \chi_{c}^{*} f(p, \vartheta, \xi)
\end{aligned}
$$

Energy conservation equation:

$$
\begin{aligned}
& \partial_{t}\left[c_{w} \varrho_{w}\left(\chi_{c}^{*} \phi_{c}(\xi)+\chi_{a}^{*} \phi_{a}\right) \mathcal{S}(p) \vartheta\right] \\
& \partial_{t}\left[\left(\left[\chi_{c}^{*} \varrho_{s c} c_{s c}\left(1-\phi_{c}(\xi)\right)+\chi_{a}^{*} \varrho_{s a} c_{s a}\left(1-\phi_{a}\right)\right]\right) \vartheta\right] \\
& -\nabla \cdot\left[\boldsymbol{\Lambda}^{*}(p, \vartheta, \xi) \nabla \vartheta+c_{w} \vartheta \boldsymbol{A}^{*}(p, \vartheta, \xi) \nabla p\right] \\
= & \alpha_{2} \chi_{c}^{*} f(p, \vartheta, \xi) .
\end{aligned}
$$

The system is coupled with the integral condition

$$
\xi(x, t)=\int_{0}^{t} f(p(x, s), \vartheta(x, s), \xi(x, s)) \mathrm{d} s .
$$

In (5) and (6), the homogenized coefficient functions are given by

$$
\chi_{c}^{*}=\int_{\mathcal{Y}} \chi_{c}(y) \mathrm{d} y, \quad \chi_{a}^{*}=\int_{\mathcal{Y}} \chi_{a}(y) \mathrm{d} y,
$$




$$
\begin{array}{r}
\Lambda_{i j}^{*}(\omega, \eta, \zeta)=\int_{\mathcal{Y}} \underbrace{\left(\chi_{c}(y) \lambda_{c}(\omega, \eta, \zeta)+\chi_{a}(y) \lambda_{a}(\omega, \eta)\right)}_{\lambda(y, \omega, \eta, \zeta)} \times \\
\times\left(\delta_{i j}+\delta_{i k} \frac{\partial v_{j}}{\partial y_{k}}\right) \mathrm{d} y
\end{array}
$$

and

$$
\begin{array}{r}
A_{i j}^{*}(\omega, \eta, \zeta)=\int_{\mathcal{Y}} \underbrace{\frac{\varrho_{w} k_{R}(\mathcal{S}(\omega))}{\mu(\eta)}\left[\chi_{c}(y) k_{c}(\zeta)+\chi_{a}(y) k_{a}\right]}_{a(y, \omega, \eta, \zeta)} \times \\
\times\left(\delta_{i j}+\delta_{i k} \frac{\partial w_{j}}{\partial y_{k}}\right) \mathrm{d} y, \quad(9)
\end{array}
$$

where $w_{i}$ and $v_{i} \in W_{\text {per }}^{1,2}(\mathcal{Y}), i=1,2$, are periodic solutions (in a weak sense) of the following cell problems, respectively,

$$
-\nabla_{y} \cdot\left(a(y, \omega, \eta, \zeta)\left(\boldsymbol{e}_{i}+\nabla_{y} w_{i}\right)\right)=0 \text { in } \mathcal{Y}
$$

and

$$
-\nabla_{y} \cdot\left(\lambda(y, \omega, \eta, \zeta)\left(\boldsymbol{e}_{i}+\nabla_{y} v_{i}\right)\right)=0 \text { in } \mathcal{Y}
$$

Here, a summation convention is used, i.e. summation is performed over repeated indices. Further, $\delta_{i j}$ (or $\left.\delta_{i k}\right)$ denotes the Kronecker delta and $\left\{\boldsymbol{e}_{1}, \boldsymbol{e}_{2}\right\}$ is the canonical basis of $\mathbb{R}^{2}$. Note that the problems 10 and (11), respectively, define $w_{i}$ and $v_{i}, i=1,2$, up to an additive constants. Usually, we choose $w_{i}$ and $v_{i}$ such that

$$
\int_{\mathcal{Y}} w_{i} \mathrm{~d} y=0 \quad \text { and } \quad \int_{\mathcal{Y}} v_{i} \mathrm{~d} y=0 .
$$

It is easily seen that the homogenized coefficients (8) and $(9)$ do not depend on the choice of the additive constant.

\section{Numerical SOlution}

\subsection{Macroscopic FE Formulation}

Let $0=t_{0}<t_{1}<\cdots<t_{N}=T$ be an equidistant partitioning of time interval $[0, T]$ with the discrete time step $\Delta t,\left\{t_{n}\right\}_{n=0}^{N}, \Delta t=T / N$. For any function $\zeta$, we will use the approximation $\zeta_{n} \approx \zeta\left(t_{n}\right)$ and denote the backward time difference as $\delta_{\Delta t}\left[\zeta_{n+1}\right]$, where $\delta_{\Delta t}\left[\zeta_{n+1}\right]:=\frac{\zeta_{n+1}-\zeta_{n}}{\Delta t} \approx \frac{d \zeta\left(t_{n+1}\right)}{d t}$. By $\mathcal{T}_{h}$, we denote an admissible quadrilateral partition of $\Omega$ with a mesh size $h$ with standard properties from the finite element theory (see e.g. [30]). Let $\mathbb{W}_{h}$ be the standard conforming linear finite element space over $\mathcal{T}_{h}$. Let $p_{0}^{h}=$ $p_{0}, \vartheta_{0}^{h}=\vartheta_{0}$, and $\xi_{0}^{h}=0$ in $\Omega$. For $n=0, \ldots, N-1$, we seek $\left[p_{n+1}^{h}, \vartheta_{n+1}^{h}, \xi_{n+1}^{h}\right] \in \mathbb{W}_{h} \times \mathbb{W}_{h} \times C(\Omega)$ the approximate solution of $[p, \vartheta, \xi]$ at time $t_{n}$, such that

$$
\begin{aligned}
& \varrho_{w} \chi_{c}^{*} \int_{\Omega} \delta_{\Delta t}\left[\phi_{c}\left(\xi_{n+1}^{h}\right) \mathcal{S}\left(p_{n+1}^{h}\right)\right] \varphi^{h} \mathrm{~d} x \\
& +\varrho_{w} \chi_{a}^{*} \phi_{a} \int_{\Omega} \delta_{\Delta t}\left[\mathcal{S}\left(p_{n+1}^{h}\right)\right] \varphi^{h} \mathrm{~d} x \\
& +\int_{\Omega} \boldsymbol{A}^{*}\left(p_{n}^{h}, \vartheta_{n}^{h}, \xi_{n}^{h}\right) \nabla p_{n+1}^{h} \cdot \nabla \varphi^{h} \mathrm{~d} x \\
& +\int_{\partial \Omega} \beta_{c}(x) p_{n+1}^{h} \varphi^{h} \mathrm{~d} S \\
& =\alpha_{1} \chi_{c}^{*} \int_{\Omega} f\left(p_{n}^{h}, \vartheta_{n}^{h}, \xi_{n}^{h}\right) \varphi^{h} \mathrm{~d} x \\
& +\int_{\partial \Omega} \beta_{c}(x)\left(p_{\infty}\right)_{n+1} \varphi^{h} \mathrm{~d} S
\end{aligned}
$$

holds for all $\varphi^{h} \in \mathbb{W}_{h}$, further

$$
\begin{aligned}
& c_{w} \varrho_{w} \chi_{c}^{*} \int_{\Omega} \delta_{\Delta t}\left[\phi_{c}\left(\xi_{n+1}^{h}\right) \mathcal{S}\left(p_{n+1}^{h}\right) \vartheta_{n+1}^{h}\right] \psi^{h} \mathrm{~d} x \\
& +c_{w} \varrho_{w} \chi_{a}^{*} \phi_{a} \int_{\Omega} \delta_{\Delta t}\left[\mathcal{S}\left(p_{n+1}^{h}\right) \vartheta_{n+1}^{h}\right] \psi^{h} \mathrm{~d} x \\
& +\int_{\Omega} \delta_{\Delta t}\left[\chi_{c}^{*} \varrho_{s c} c_{s c}\left(1-\phi_{c}\left(\xi_{n+1}^{h}\right)\right) \vartheta_{n+1}^{h}\right] \psi^{h} \mathrm{~d} x \\
& +\int_{\Omega} \delta_{\Delta t}\left[\chi_{a}^{*} \varrho_{s a} c_{s a}\left(1-\phi_{a}\right) \vartheta_{n+1}^{h}\right] \psi^{h} \mathrm{~d} x \\
& +\int_{\Omega} \Lambda^{*}\left(p_{n}^{h}, \vartheta_{n}^{h}, \xi_{n}^{h}\right) \nabla \vartheta_{n+1}^{h} \cdot \nabla \psi^{h} \mathrm{~d} x \\
& +\int_{\Omega} c_{w} \vartheta_{n}^{h} \boldsymbol{A}^{*}\left(p_{n}^{h}, \vartheta_{n}^{h}, \xi_{n}^{h}\right) \nabla \vartheta_{n+1}^{h} \cdot \nabla \psi^{h} \mathrm{~d} x \\
& +\int_{\partial \Omega} \alpha_{c}(x) \vartheta_{n+1}^{h} \psi^{h} \mathrm{~d} S+c_{w} \int_{\partial \Omega} \beta_{c}(x) \vartheta_{n}^{h} p_{n+1}^{h} \psi^{h} \mathrm{~d} S \\
& =\alpha_{2} \chi_{c}^{*} \int_{\Omega} f\left(p_{n}^{h}, \vartheta_{n}^{h}, \xi_{n}^{h}\right) \psi^{h} \mathrm{~d} x \\
& +\int_{\partial \Omega} \alpha_{c}(x)\left(\vartheta_{\infty}\right)_{n+1} \psi^{h} \mathrm{~d} S \\
& +c_{w} \int_{\partial \Omega} \beta_{c}(x) \vartheta_{n}^{h}\left(p_{\infty}\right){ }_{n+1} \psi^{h} \mathrm{~d} S
\end{aligned}
$$

holds for all $\psi^{h} \in \mathbb{W}_{h}$ and

$$
\xi_{n+1}^{h}=\xi_{n}^{h}+\Delta t f\left(p_{n}^{h}, \vartheta_{n}^{h}, \xi_{n}^{h}\right) .
$$

The unknown vector field $\boldsymbol{x}^{n+1}=$ $\left(\boldsymbol{p}^{n+1}, \boldsymbol{\vartheta}^{n+1}, \boldsymbol{\xi}^{n+1}\right)^{T}$ is introduced, and the Galerkin procedure is applied. This leads to a system of non-linear algebraic equations

$$
\begin{aligned}
& \boldsymbol{b}\left(\boldsymbol{x}^{n+1}\right)-\boldsymbol{b}\left(\boldsymbol{x}^{n}\right)+\Delta t \mathbf{K}^{n} \boldsymbol{x}^{n+1} \\
&+\Delta t \boldsymbol{f}^{n+1}\left(\boldsymbol{x}^{n+1}\right)=\mathbf{0}
\end{aligned}
$$

where $\boldsymbol{p}^{n+1}, \boldsymbol{\vartheta}^{n+1}$, and $\boldsymbol{\xi}^{n+1}$ store the unknown nodal values of water pressure, temperature, and hydration degree at a time $t_{n+1}$, respectively. The non-linear system 16 is solved iteratively using the Newton's method. 


\subsection{PERIODIC CELL PROBLEM}

The problems 10 and 11 need to be solved in each discrete time step. For fixed $\omega, \eta$, and $\zeta \in \mathbb{R}$, both problems, 10 and 11, can be written in a general form as a periodic cell problem $(i=1,2)$

$$
-\nabla_{y} \cdot\left(A(y)\left(\boldsymbol{e}_{i}+\nabla_{y} u_{i}\right)\right)=0 \text { in } \mathcal{Y},
$$

where $A$ is a given step function,

$$
A(y):= \begin{cases}A_{c} \in \mathbb{R}_{+} & \text {for } y \in \mathcal{Y}_{c} \\ A_{a} \in \mathbb{R}_{+} & \text {for } y \in \mathcal{Y}_{a}\end{cases}
$$

and $u_{i}$ is the unknown function satisfying the periodic boundary conditions on $\partial \mathcal{Y}$.

\subsubsection{The Classical FE method}

The variational formulation of (17) reads as follows: we seek $u_{i} \in W_{\text {per }}^{1,2}(\mathcal{Y})$ such that $(i=1,2)$

$$
\int_{\mathcal{Y}} A(y) \nabla u_{i} \cdot \nabla \varphi \mathrm{d} y=-\int_{\mathcal{Y}} A(y) \boldsymbol{e}_{i} \cdot \nabla \varphi \mathrm{d} y
$$

for all $\varphi \in W_{\text {per }}^{1,2}(\mathcal{Y})$. A finite element approximation is obtained by restricting the weak formulation (19) to a finite dimensional subspace $W_{\text {per, }}^{1,2}(\mathcal{Y})$ of $W_{\text {per }}^{1,2}(\mathcal{Y})$. Then, we seek the approximate solution $u_{i}^{h} \in W_{p e r, h}^{1,2}(\mathcal{Y})$ satisfying the equation

$$
\int_{\mathcal{Y}} A(y) \nabla u_{i}^{h} \cdot \nabla \varphi^{h} \mathrm{~d} y=-\int_{\mathcal{Y}} A(y) \boldsymbol{e}_{i} \cdot \nabla \varphi^{h} \mathrm{~d} y
$$

for all $\varphi^{h} \in W_{p e r, h}^{1,2}(\mathcal{Y})$.

\subsubsection{Computation of the homogenized COEFFICIENTS BY AN ANALYTICAL APPROXIMATION}

It is worth pointing out that the cell problems 10 and (11) need to be solved at each integration point and in each discrete time step $n=0,1, \ldots, N$ of the problem $13-(15)$. This means that a very large number of cell problems need to be solved in order to compute homogenized coefficients $\boldsymbol{A}^{*}$ and $\boldsymbol{\Lambda}^{*}$ in the whole macroscopic domain $\Omega$.

In [20, the authors proposed an analytical approximation of the solution to the periodic cell problem (17) with a step function $A$ defined by 18 . It can be easily shown that the functions

$$
\tilde{u}_{1}(y)=\int_{0}^{y_{1}} \frac{d s}{A\left(s, y_{2}\right)}\left(\int_{0}^{1} \frac{d s}{A\left(s, y_{2}\right)}\right)^{-1}-y_{1}
$$

and

$$
\tilde{u}_{2}(y)=\int_{0}^{y_{2}} \frac{d s}{A\left(y_{1}, s\right)}\left(\int_{0}^{1} \frac{d s}{A\left(y_{1}, s\right)}\right)^{-1}-y_{2}
$$

satisfy the equation (17) almost everywhere in $\mathcal{Y}$, see 20, Theorem 2.1]. The proposed solution can be seen as an approximation to the solution of $\sqrt{19}$ in $L^{2}(\mathcal{Y})$. The computational cost savings are evident because of the closed-form approximation of the solution to the periodic cell problem.

It is worth noting that, for the aggregate of a rectangular shape symmetrically placed within the periodic cell, which is the case considered in the following numerical experiments, the aforementioned approximation leads to simple formulae for the homogenized coefficients, see also [20] 23. For $\boldsymbol{\Lambda}^{*}$ (and for $\boldsymbol{A}^{*}$ analogously), we can write

$$
\begin{aligned}
\Lambda_{11}^{*}\left(p_{k}, \vartheta_{k}, \xi_{k}\right) & =2 d_{2} \lambda_{c}\left(p_{k}, \vartheta_{k}, \xi_{k}\right) \\
+ & \frac{1-2 d_{2}}{\frac{2 d_{1}}{\lambda_{c}\left(p_{k}, \vartheta_{k}, \xi_{k}\right)}+\frac{1-2 d_{1}}{\lambda_{a}\left(p_{k}, \vartheta_{k}\right)}}, \\
\Lambda_{22}^{*}\left(p_{k}, \vartheta_{k}, \xi_{k}\right) & =2 d_{1} \lambda_{c}\left(p_{k}, \vartheta_{k}, \xi_{k}\right) \\
+ & \frac{1-2 d_{1}}{\frac{2 d_{2}}{\lambda_{c}\left(p_{k}, \vartheta_{k}, \xi_{k}\right)}+\frac{1-2 d_{2}}{\lambda_{a}\left(p_{k}, \vartheta_{k}\right)}},
\end{aligned}
$$

and

$$
\Lambda_{12}^{*}=\Lambda_{21}^{*}=0,
$$

where $p_{k}, \vartheta_{k}$, and $\xi_{k}$, respectively, are the water pressure, temperature, and hydration degree at the $k$-th integration point of the macroscopic (homogenized) model, and $d_{1}$ and $d_{2}$ are the geometrical parameters of the periodic cell, see Figure 1 .

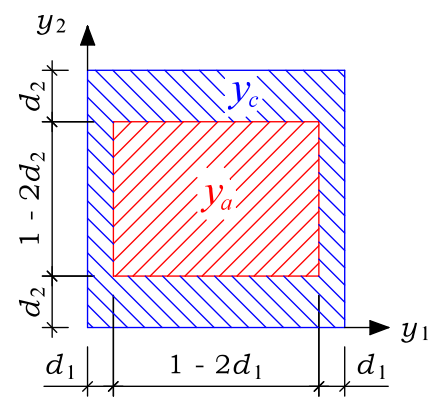

Figure 1. Cell geometry.

The solution of the cell problem in $\mathcal{Y}$ is crucial for obtaining the homogenized coefficients in the global domain $\Omega$. Therefore, a comparison of solutions of the homogenized problem (13)- 15), with homogenized coefficients obtained by using the analytical formulae (23) - 25 and the one computed numerically according to 201 , is one of the major goals of the present work, see the next section.

\section{NUMERICAL EXPERIMENTS}

The mesoscale model and the homogenization framework have both been implemented in a MATLAB [31] code, which is employed for the following numerical experiments. For the spatial discretization, the finite element method is utilized by using four-node bilinear elements with $3 \times 3$ integration points. The temporal discretization is performed by a semi-implicit difference scheme, see Section 4 . 
The aim of the illustrative examples is to demonstrate some of the properties of the mesoscale model and the homogenization framework by analysing the effects of changing (i) the characteristic length $\varepsilon$, (ii) the aggregate shape, (iii) the FE mesh size, and (iv) the approach to solving the local problem.

For the numerical experiments presented in this paper, the cell geometry shown in Figure 1 is assumed. The rectangular aggregate shape was chosen for the reason that it can be easily analysed by the analytical approach and it can be simply discretized by fournode bilinear finite elements. Similar examples have also been investigated by other researches focusing on the homogenization problem, e.g. [19 23].

For real-world problems, the cell geometry can be modified in order to represent the material more accurately. Concrete is a cementitious composite formed by the aggregates of different shapes and sizes. This could be implemented in the present models by the appropriate setting of the periodic cell geometry. Within the cell, multiple domains representing the aggregates can be modelled, each of them of a different shape and size, and the local problem can be solved by the finite element method. Due to the limited scope of the paper, such problem is not presented and will be analysed in our future work.

All the material parameters and properties of concrete components adopted for the calculations as well as the parameters of initial and boundary conditions are summarized in Section 7

\subsection{Convergence AnAlysis - LAYERED STRUCTURE}

The first example is focused on an analysis of hydration and transport processes in a structure (wall) formed by parallel layers of a fresh cement paste and aggregates. The objective of this hypothetical example is to show the convergence of the mesoscale solution towards the homogenized problem solution, as described for similar problems, e.g., in [19 23].

This type of example has been chosen as in this case (a layered structure), the homogenized coefficients can be obtained by an exact solution, see [23, p. 2262], cf. e.g. [26].

The geometrical parameters of the analysed problem are depicted in Figure2

The calculations are performed on the mesoscale level for three different values of $\varepsilon$ : $0.1,0.05,0.01$. In all the cases, we use the same finite element mesh of 500 elements in total with $\Delta x=1 \mathrm{~mm}$, see Figure 2, The homogenized problem is solved twice. Once with the same mesh as for the mesoscale level $(\Delta x=1 \mathrm{~mm})$, once with a coarse mesh with $\Delta x=25 \mathrm{~mm}$. The time step is set to $\Delta t=30 \mathrm{~s}$ in all cases.

The obtained distributions of water pressure across the analysed structure are displayed in Figure 3 The temperature profiles are depicted in Figure 4 The distributions of hydration degree are shown in Figure 5.



Figure 2. Scheme of the analysed problem, finite element (FE) mesh, and boundary conditions (BC).

For the detailed convergence analysis, we focus on the resulting distributions of temperature and water pressure. In Tables 1 and 2 the differences between the temperatures and water pressures determined by the mesoscale $\left(\vartheta^{\varepsilon}, p^{\varepsilon}\right)$ and homogenized $(\vartheta, p)$ models for selected times are illustrated by the respective $L^{2}$ error norms, see [19 23].

\begin{tabular}{lccc}
\hline \multirow{\varepsilon}{*}{$\varepsilon$} & \multicolumn{3}{c}{$\left\|\vartheta^{\varepsilon}-\vartheta\right\|_{2}(\mathrm{Km})$} \\
\cline { 2 - 4 } & $t=8 \mathrm{~h}$ & $t=16 \mathrm{~h}$ & $t=24 \mathrm{~h}$ \\
\hline 0.1 & $8.9 \times 10^{-3}$ & $5.8 \times 10^{-3}$ & $2.4 \times 10^{-3}$ \\
0.05 & $2.2 \times 10^{-3}$ & $1.5 \times 10^{-3}$ & $7.6 \times 10^{-4}$ \\
0.01 & $8.3 \times 10^{-5}$ & $7.9 \times 10^{-5}$ & $1.0 \times 10^{-4}$ \\
\hline
\end{tabular}

TABLE 1. Error norms for the temperature determined by the mesoscale and homogenized models for selected times.

\begin{tabular}{lrrr}
\hline \multirow{2}{*}{$\varepsilon$} & \multicolumn{3}{c}{$\left\|p^{\varepsilon}-p\right\|_{2}(\mathrm{Pam})$} \\
\cline { 2 - 4 } & $t=8 \mathrm{~h}$ & $t=16 \mathrm{~h}$ & \multicolumn{1}{c}{$t=24 \mathrm{~h}$} \\
\hline 0.1 & 2560.0 & 1520.0 & 819.8 \\
0.05 & 734.9 & 358.4 & 197.9 \\
0.01 & 29.9 & 14.5 & 9.3 \\
\hline
\end{tabular}

TABLE 2. Error norms for the water pressure determined by the mesoscale and homogenized models for selected times.

From Figures 43 and Tables 1 and 2 it is clear that with the decreasing value of $\varepsilon$, the mesoscale solution converges towards the homogenized problem solution. Moreover, it can be seen that for the homogenized problem, a coarse finite element mesh provides almost the same results as a fine mesh.

\subsection{Illustrative EXAMPLE - CONCRETE COLUMN CROSS-SECTION}

In the second example, the hydration and transport processes in a concrete column of a square crosssection are simulated. The objective of the example is to illustrate the usage of the numerical procedures 

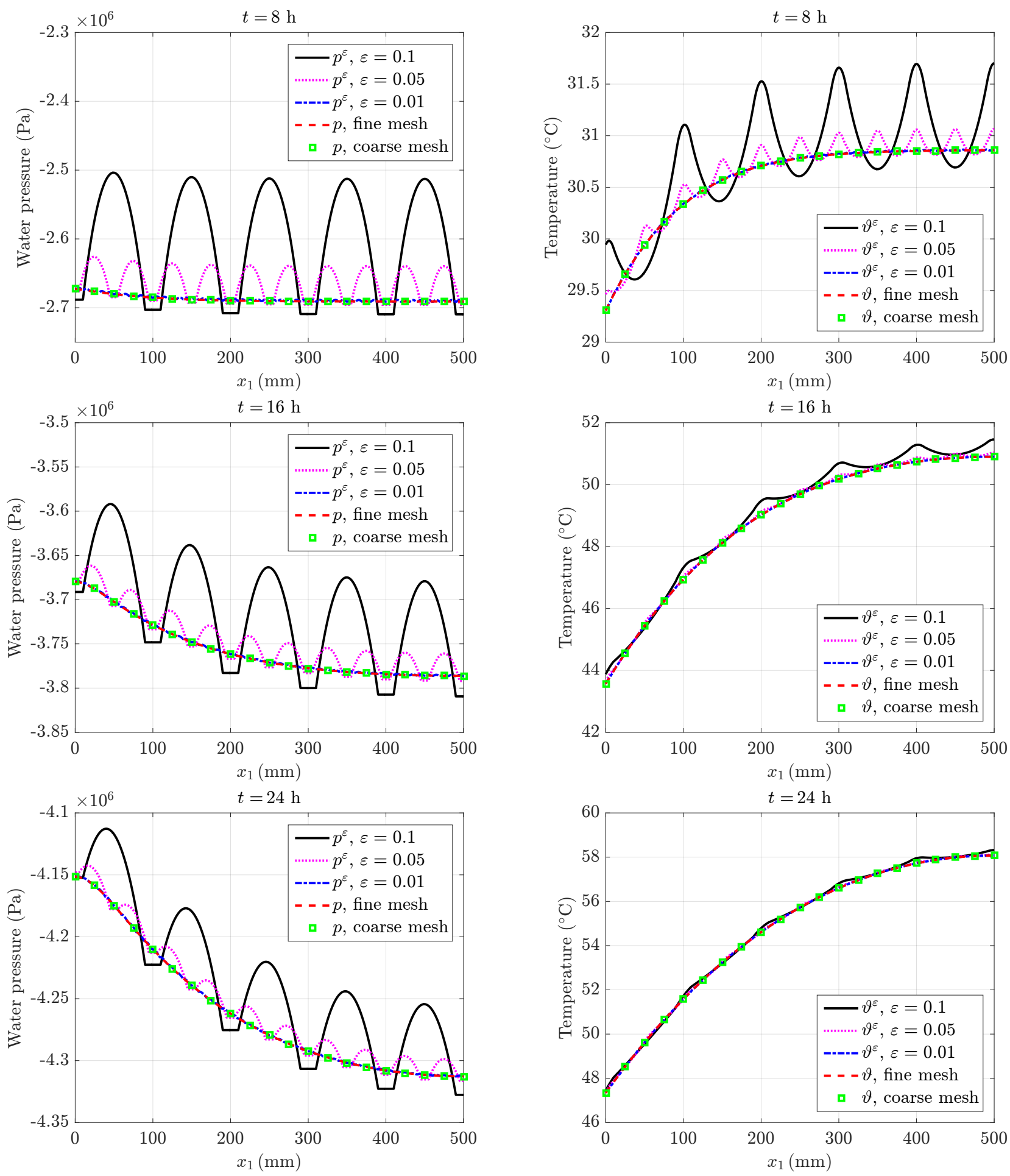

FigURE 3. Distribution of water pressure across the analysed structure.

for the determination of the homogenized material parameters, as described in Section 4.2. The scheme of the analysed problem is depicted in Figure 6

As can be seen from Figure 6, we assume two variants of the aggregate shape: square (denoted as Variant $\mathrm{S}$ ) and rectangle (denoted as Variant R).

On the mesoscale level, the simulations are performed for $\varepsilon=0.025$, i.e. with $8 \times 8$ cells for the analysed section. In both cases (Variants S and R),

FiguRE 4. Distribution of temperature across the analysed structure.

we use the same finite element mesh of square elements of the size of $2.5 \mathrm{~mm}$ (6400 elements in total), see Figure 7

For the homogenized model, a finite element mesh of $10 \times 10$ square elements is used on the macroscale level (element size of $20 \mathrm{~mm}$ ). The homogenized material parameters are determined using two different approaches (see Section 4.2): (i) using the finite element solution of the periodic cell problem with the 

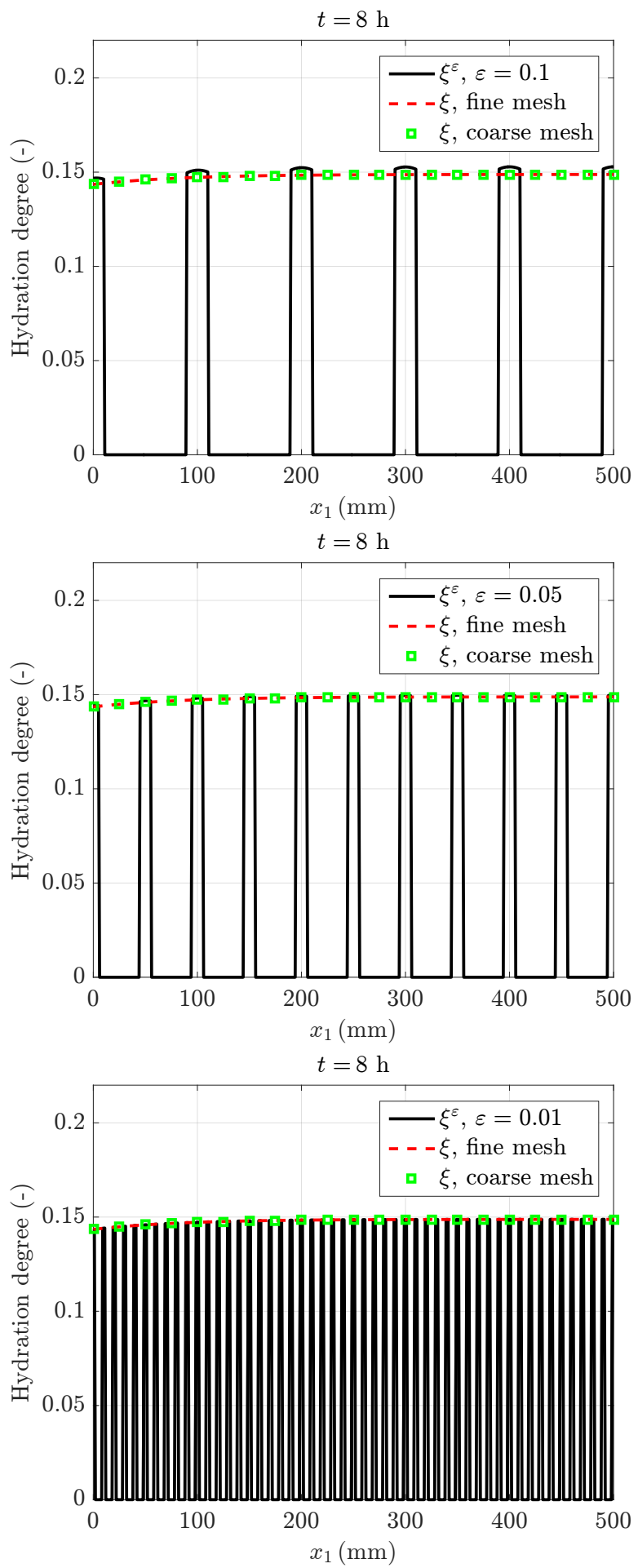

FIGURE 5. Distribution of hydration degree across the analysed structure.

mesh of $10 \times 10$ square elements for one quarter of the cell (element size of $50 \mathrm{~mm}$; due to symmetry, only one quarter of the cell is modelled), and (ii) using the simplified approach proposed by Sviercoski et al. [20]. In the following figures, the results obtained by the homogenized model using these two approaches are indexed as (FE) and (Approx.), respectively.

The time step is set to $\Delta t=60 \mathrm{~s}$ in all the cases.

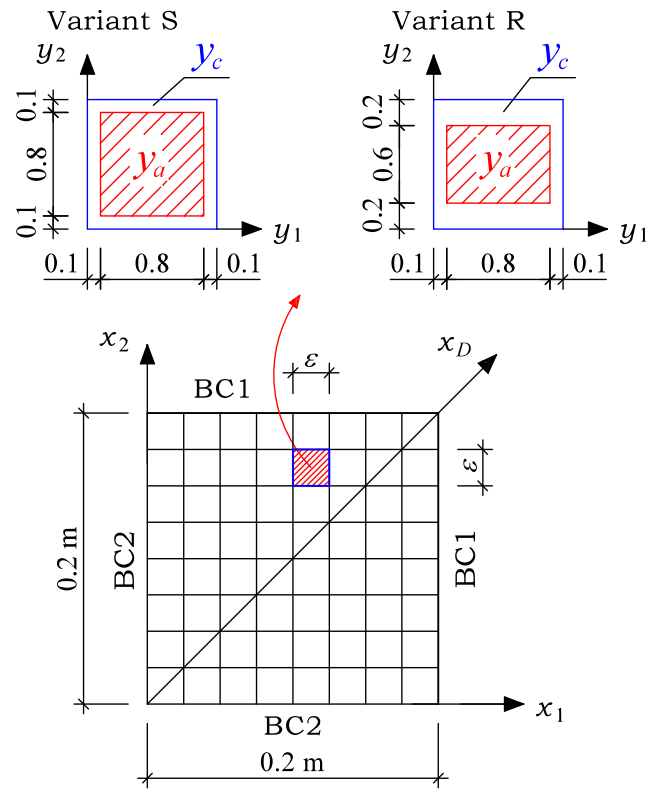

Figure 6 . Scheme of the analysed problem, cell geometries, boundary conditions (BC).


Figure 7. Finite element mesh used for the mesoscale simulations: Variant S (left), Variant R (right).

The obtained profiles of water pressure, temperature, and hydration degree along the diagonal of the analysed section $\left(x_{D}\right.$, see Figure 6, see also e.g. [19]) are displayed in Figure 8

A more detailed comparison of the temperature distribution is presented using the isolines maps (contour plots) in Figure 9, cf. e.g. [21 23].

For illustration, selected results (hydration degree and saturation) are shown using the filled contour maps in Figures 10 and 11] cf. e.g. [23].

As can be seen, the presented homogenized model provides a sufficiently accurate approximation to the mesoscale problem of hydration and transport processes in early age concrete. This holds true mainly for the temperature, which is, from the engineering point of view, the most important variable.

The analysed example also indicates that, in comparison with the classical FE-approach, the approximate solution of the periodic cell problem (determination of the homogenized material coefficients) proposed by Sviercoski et al. 20] gives almost the same results while achieving significant computational cost savings. 
The computational cost savings are evident because of the closed-form approximation of the solution to the periodic cell problem, which needs to solved at each integration point (at the macroscale) and in each discrete time step. By the analytical solution, the homogenized coefficients are obtained directly by a closed-form formulae - see formulae 23 - 25 , while for the finite element approach, the local problem needs to be solved numerically.

\section{Conclusions}

In the paper, we have proposed a fully coupled twoscale (meso-macro) homogenization framework (theoretically investigated in [17, 29]) for the modelling of simultaneous heat transfer, moisture flows and hydration phenomena in fresh concrete.

A modified mesoscale model has been introduced by assuming concrete as a composite material with two periodically distributed mesoscale components, cement paste and aggregates, partially saturated with liquid water and gas.

For the mesoscale problem, an upscaling method has been utilized in order to obtain the homogenized model. The homogenized coefficients have been determined based on the solution of the periodic cell problem.

Two different approaches have been adopted for solving the periodic cell problem: (i) the classical finite element method, (ii) a simplified approach based on an analytical approximation proposed by Sviercoski et al. 20.

The resulting algorithm, based on the finite element discretization in space and a semi-implicit finite difference discretization in time, has been implemented in MATLAB environment and employed for several numerical experiments.

The results of the presented examples have confirmed the following.

- With a decreasing value of the characteristic length $\varepsilon$, representing the small scale variability of the concrete composite, the mesoscale solution converges towards the homogenized problem solution.

- For the homogenized problem, a coarse finite element mesh provides almost the same results as the fine mesh.

- The presented homogenized model provides a sufficiently accurate approximation to the mesoscale problem of hydration and transport processes in early age concrete.

- For the homogenized model, the approximate solution of the periodic cell problem (determination of the homogenized material coefficients) proposed by Sviercoski et al. 20] gives almost the same results as the classical FE approach while achieving significant computational cost savings.

In our future work, we will focus on:
- numerical experiments investigating the effect of the cell geometry (aggregates of different shapes and sizes);

- utilization of the homogenization approach for the analysis of transport processes in concrete exposed to high temperatures.

\section{Appendix - Model PARAmeters}

Based on a literature review, the model parameters employed for the numerical experiments presented in this paper have been adopted as follows (see also our previous work [18]).

The values of density and heat capacity of concrete components are taken as: $\varrho_{w}=1000 \mathrm{~kg} \mathrm{~m}^{-3}$, $\varrho_{s a}=2830 \mathrm{~kg} \mathrm{~m}^{-3}, \varrho_{s c}=3220 \mathrm{~kg} \mathrm{~m}^{-3}, c_{w}=$ $4180 \mathrm{~J} \mathrm{~kg}^{-1} \mathrm{~K}^{-1}, \quad c_{s a}=840 \mathrm{~J} \mathrm{~kg}^{-1} \mathrm{~K}^{-1}, \quad c_{s c}=$ $750 \mathrm{~J} \mathrm{~kg}^{-1} \mathrm{~K}^{-1}$ [16, Table 1], [32, Example 13.1].

The porosity of cement paste, $\phi_{c}(\xi)$, is determined by [4, (29)], with $\phi_{c, \infty}=0.2$, and $A_{\phi}=0.35$ [4, Fig. 1]. The porosity of aggregate, $\phi_{a}$, is set to a constant value $\phi_{a}=0.05$, see e.g. 33 .

The intrinsic permeability of cement paste, $k_{c}(\xi)$, is given by [4, (32)], with $k_{c, \infty}=10^{-18} \mathrm{~m}^{2}$ [4, Table I], [32, p. 308] and $A_{k}=8$ [4, p. 312]. The intrinsic permeability of aggregate, $k_{a}$, is set to a constant value $k_{a}=10^{-20} \mathrm{~m}^{2}$, see e.g. 33 .

The thermal conductivity of cement paste and aggregate, $\lambda_{c}(p, \xi)$ and $\lambda_{a}(p)$, respectively, is determined by [4, (37)] with the constant thermal conductivity of a dry material, namely $\lambda_{c, d r y}=1.0 \mathrm{~W} \mathrm{~m}^{-1} \mathrm{~K}^{-1}$ and $\lambda_{a, d r y}=2.4 \mathrm{~W} \mathrm{~m}^{-1} \mathrm{~K}^{-1}[16$, p. 136, Table 1].

The degree of the saturation with liquid water, $\mathcal{S}$, is taken from [34, (20)] with the material parameters specified in [34, Table 5] for ordinary concrete $(a=$ $18.6237 \mathrm{MPa}, b=2.2748)$. Here, we follow a simplified assumption that the capillary pressure, $p_{c}$, and the liquid water pressure, $p$, are in the relation $p_{c}=$ $p_{\text {atm }}-p$ with $p_{\text {atm }}$ being the atmospheric pressure [4] pp. 305-306], [13, Chapter 4.2.2].

The relative hydraulic conductivity, $k_{R}(\mathcal{S})$, and the dynamic viscosity, $\mu(\vartheta)$, respectively, are adopted from [34, (21)], [35, (38)], and [35. (AI.8)].

The hydration degree rate $f$ is determined as (e.g. [4, (23)], [16, (7)], [32, (13.31)-(13.35)])

$$
f=A(\xi) \beta_{\vartheta}(\vartheta) \beta_{R H}(R H(p, \vartheta)),
$$

where the formulae and most of the material parameters for $A(\xi), \beta_{\vartheta}(\vartheta)$, and $\beta_{R H}(R H(p, \vartheta)$, are adopted from 16 , 32 with: $B_{1}=23.4 /(24 \times 3600) \mathrm{s}^{-1}$, $B_{2}=7 \times 10^{-4}, \eta=6.7, Q_{e} / R=4600 \mathrm{~K}$, and $\alpha_{e}=7.5$. The notation is taken from 32 . The relative humidity, $R H$, is calculated from [4, (12)], by assuming $p_{c}=p_{a t m}-p$ (see above). The ultimate hydration degree, $\xi_{\infty}$, is set to $\xi_{\infty}=0.8$ (see [2, (13)], [16, $(9),(10)])$.

The constants $\alpha_{1}$ and $\alpha_{2}$ in the source terms in equations (1), (2), or (5), (6), respectively, are assumed 

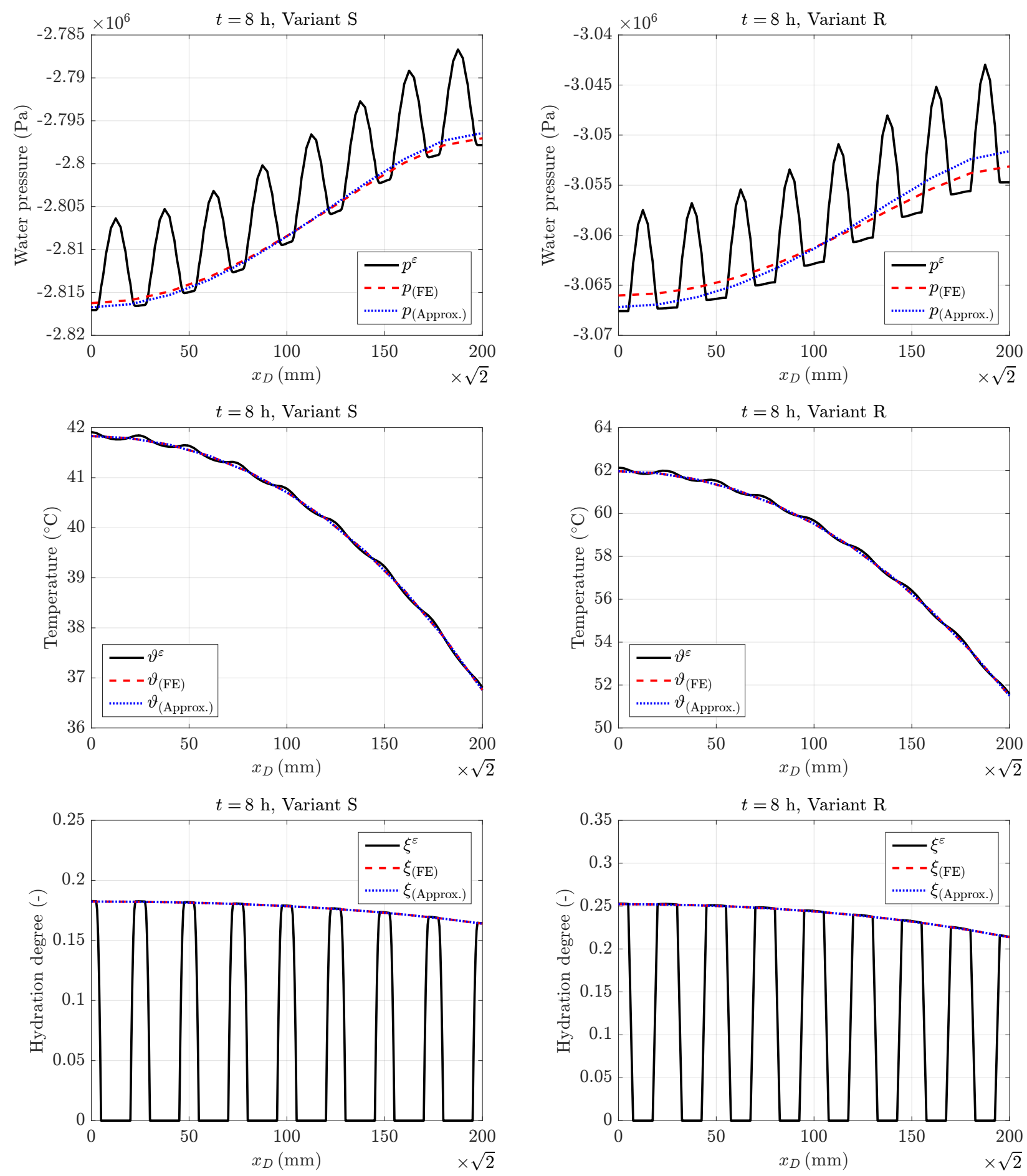

FiguRE 8. Water pressure, temperature, and hydration degree profiles along the diagonal of the analysed section.

as $([4,16,32$, see also [29] and references therein)

$$
\begin{aligned}
& \alpha_{1}=-Q_{w, p o t} m_{c}, \\
& \alpha_{2}=Q_{h, p o t} m_{c},
\end{aligned}
$$

with $Q_{w, p o t}=0.25, Q_{h, p o t}=510 \times 10^{3} \mathrm{Jkg}^{-1}$, and $m_{c}=\left(1-\phi_{c}(0)\right) \varrho_{s c}$.

The initial conditions are set to $p_{0}=-2.155 \times$ $10^{6} \mathrm{~Pa}\left(\mathcal{S}_{0}=0.99\right)$ and $\vartheta_{0}=293.15 \mathrm{~K}$.

The parameters of the boundary conditions are taken as: $\beta_{c}=10^{-30} \mathrm{sm}^{-1}, p_{\infty}=-6.9 \times 10^{7} \mathrm{~Pa}$
$\left(R H_{\infty}=0.6\right), \alpha_{c}=4 \mathrm{~W} \mathrm{~m}^{-2} \mathrm{~K}^{-1}$, and $\vartheta_{\infty}=$ $293.15 \mathrm{~K}$, for the boundaries denoted as BC1 in Section 5. The boundaries denoted as BC2 in Section 5 are assumed to be perfectly insulated, i.e. $\beta_{c}=0$ and $\alpha_{c}=0$.

\section{LIST OF SYMBOLS}

$c_{w}$ Isobaric heat capacity of water $\left[\mathrm{J} \mathrm{kg}^{-1} \mathrm{~K}^{-1}\right]$

$c_{s a}$ Isobaric heat capacity of aggregates $\left[\mathrm{J} \mathrm{kg}^{-1} \mathrm{~K}^{-1}\right]$

$c_{s c}$ Isobaric heat capacity of cement paste $\left[\mathrm{J} \mathrm{kg}^{-1} \mathrm{~K}^{-1}\right]$ 



FiguRE 9. Distribution of temperature in the analysed cross-section.

$k_{a} \quad$ Intrinsic permeability of aggregates $\left[\mathrm{m}^{2}\right]$

$k_{c} \quad$ Intrinsic permeability of cement paste $\left[\mathrm{m}^{2}\right]$

$k_{c, \infty}$ Intrinsic permeability of the matured concrete $\left[\mathrm{m}^{2}\right]$

$k_{R}$ Relative permeability of liquid phase [-]

$m_{c}$ Mass of cement $\left[\mathrm{kg} \mathrm{m}^{-3}\right]$

$p$ Liquid pressure $[\mathrm{Pa}]$

$p_{0} \quad$ Initial value of liquid pressure $[\mathrm{Pa}]$

$p_{\infty} \quad$ External liquid pressure $[\mathrm{Pa}]$

$p_{\text {atm }}$ Atmospheric pressure $[\mathrm{Pa}]$

$p_{c} \quad$ Capillary pressure $[\mathrm{Pa}]$

$Q_{h, p o t}$ Potential hydration heat $\left[\mathrm{J} \mathrm{kg}^{-1}\right]$

$Q_{w, p o t}$ Chemically bound water $\left[\mathrm{kg} \mathrm{kg}^{-1}\right]$

RH Relative humidity [-]

$R H_{\infty}$ External relative humidity [-]

$\mathcal{S}$ Degree of saturation with liquid water [-]

$\mathcal{S}_{0} \quad$ Initial degree of saturation with liquid water $[-]$

\section{Greek symbols}

$\alpha_{c}$ Convective heat transfer coefficient $\left[\mathrm{W} \mathrm{m}^{-2} \mathrm{~K}^{-1}\right]$

$\beta_{c}$ Convective mass transfer coefficient $\left[\mathrm{m} \mathrm{s}^{-1}\right]$

$\delta_{i j} \quad$ Kronecker delta $[-]$

$\varepsilon \quad$ Scale parameter (characteristic length) $[-]$

$\vartheta$ Temperature $[\mathrm{K}]$

$\vartheta_{0} \quad$ Initial value of temperature $[\mathrm{K}]$

$\vartheta_{\infty} \quad$ External temperature $[\mathrm{K}]$

$\lambda_{c}$ Thermal conductivity of cement paste $\left[\mathrm{W} \mathrm{m}^{-1} \mathrm{~K}^{-1}\right]$

$\lambda_{c, d r y}$ Thermal conductivity of dry cement paste $\left[\mathrm{W} \mathrm{m}^{-1} \mathrm{~K}^{-1}\right]$

$\lambda_{a}$ Thermal conductivity of aggregates $\left[\mathrm{W} \mathrm{m}^{-1} \mathrm{~K}^{-1}\right]$

$\lambda_{a, d r y}$ Thermal conductivity of dry aggregates $\left[\mathrm{W} \mathrm{m}^{-1} \mathrm{~K}^{-1}\right.$ ]

$\mu$ Water viscosity [Pas]

$\xi \quad$ Degree of hydration [-]

$\xi_{\infty} \quad$ Final degree of hydration $[-]$ 

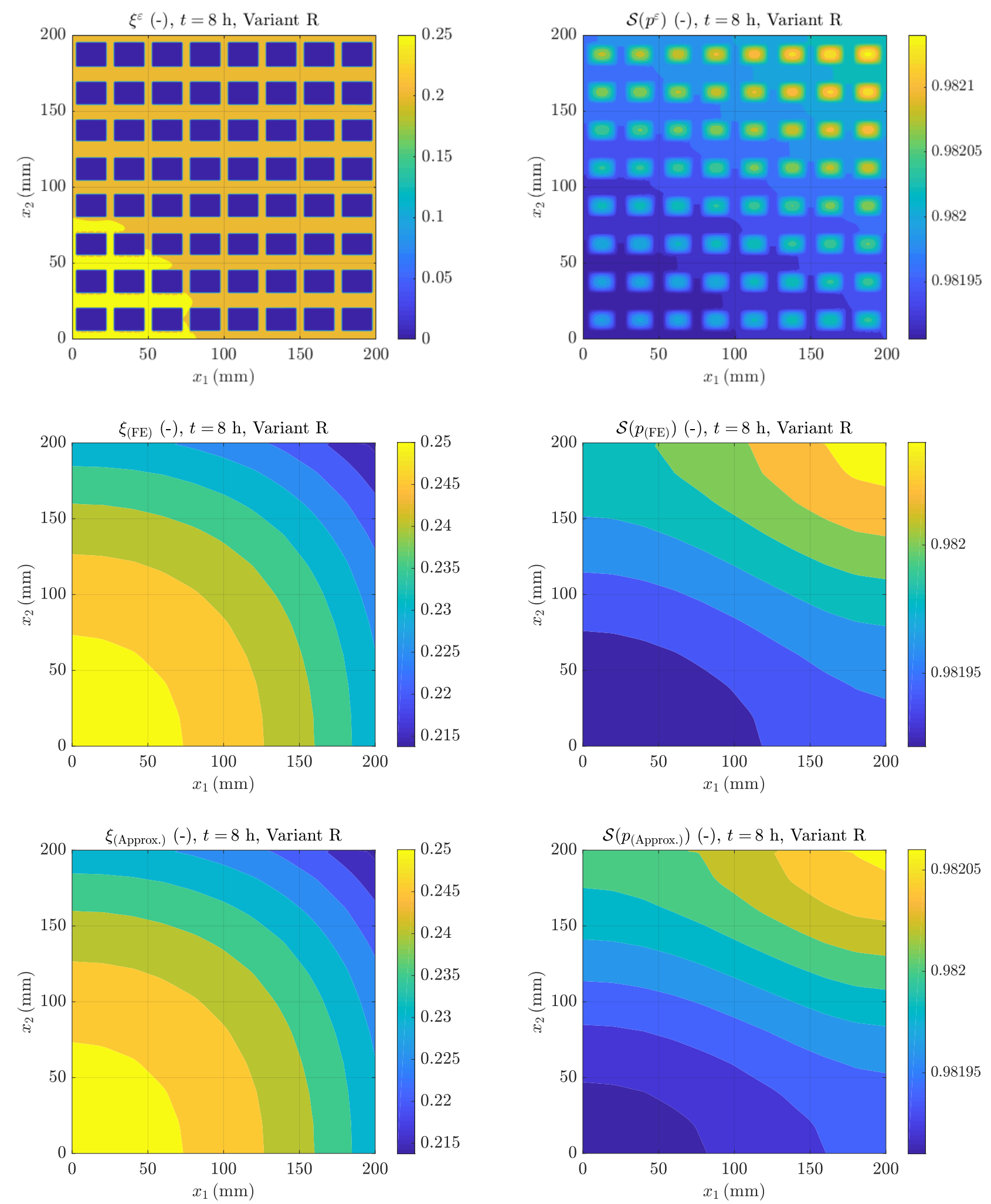

Figure 10. Distribution of hydration degree in the analysed cross-section.

FIGURE 11. Distribution of saturation degree in the analysed cross-section.

$\varrho_{w} \quad$ Liquid density $\left[\mathrm{kg} \mathrm{m}^{-3}\right]$

$\varrho_{s a}$ Density of aggregates $\left[\mathrm{kg} \mathrm{m}^{-3}\right]$

$\varrho_{s c}$ Density of cement paste $\left[\mathrm{kg} \mathrm{m}^{-3}\right]$

$\phi_{c} \quad$ Porosity of cement paste [-]

$\phi_{c, \infty}$ Porosity at final stage of hydration [-]

$\phi_{a} \quad$ Porosity of aggregates [-]

$\chi_{a}$ Characteristic function of aggregates [-]

$\chi_{c}$ Characteristic function of cement paste [-] 


\section{ACKNOWLEDGEMENTS}

The first author has been financially supported by the European Regional Development Fund (project No. CZ.02.1.01/0.0/0.0/16_019/0000778) within activities of the Center of Advanced Applied Sciences (CAAS).

The second author has been supported by the Czech Science Foundation, project no. GA17-23067S. The support is gratefully acknowledged.

\section{REFERENCES}

[1] F. J. Ulm, O. Coussy. Modeling of thermochemomechanical couplings of concrete at early ages. J Eng Mech 121(7):785-794, 1995. DOI:10.1061/(ASCE)0733-9399(1995)121:7(785)

[2] M. Cervera, J. Oliver, T. Prato.

Thermo-Chemo-Mechanical Model for Concrete. I: Hydration and Aging. J Eng Mech 125(9):1018-1027, 1999. DOI:10.1061/(ASCE)0733-9399(1999)125:9(1018).

[3] M. Cervera, R. Faria, J. Oliver, T. Prato. Numerical modelling of concrete curing, regarding hydration and temperature phenomena. Comput Struct 80(18):15111521, 2002. DOI:10.1016/S0045-7949(02)00104-9

[4] D. Gawin, F. Pesavento, B. A. Schrefler.

Hygro-thermo-chemo-mechanical modelling of concrete at early ages and beyond. Part I: hydration and hygro-thermal phenomena. Int J Numer Meth Eng 67(3):299-331, 2006. DOI:10.1002/nme.1615

[5] G. Di Luzio, G. Cusatis. Hygro-thermo-chemical modeling of high performance concrete. i: Theory. Cement Concrete Comp 31(5):301-308, 2009. DOI:10.1016/j.cemconcomp.2009.02.015

[6] B. Klemczak, M. Batog. Heat of hydration of lowclinker cements. J Therm Anal Calorim 123(2):13511360, 2016. DOI:10.1007/s10973-015-4782-y

[7] E. M. R. Fairbairn, M. Azenha (eds.). Thermal Cracking of Massive Concrete Structures: State of the Art Report of the RILEM Technical Committee 254CMS. Springer, 2019. DOI:10.1007/978-3-319-76617-1.

[8] G. Xotta, G. Mazzucco, V. A. Salomoni, et al. Composite behavior of concrete materials under high temperatures. Int J Solid Struct 64-65:86-99, 2015. DOI:10.1016/j.ijsolstr.2015.03.016

[9] J. Bear. Dynamics of Fluids in Porous Media. Courier Corporation, 1972.

[10] M. Hassanizadeh, W. G. Gray. General conservation equations for multi-phase systems: 1. Averaging procedure. Adv Water Resour 2:131-144, 1979. DOI:10.1016/0309-1708(79)90025-3

[11] M. Hassanizadeh, W. G. Gray. General conservation equations for multi-phase systems: 2. Mass, momenta, energy, and entropy equations. Adv Water Resour 2:191-203, 1979. DOI:10.1016/0309-1708(79)90035-6

[12] M. Hassanizadeh, W. G. Gray. General conservation equations for multi-phase systems: 3. Constitutive theory for porous media flow. Adv Water Resour 3(1):25-40, 1980. DOI:10.1016/0309-1708(80)90016-0

[13] K. Maekawa, R. Chaube, T. Kishi. Modelling of concrete performance: Hydration, microstructure formation, and mass transport. E \& FN Spon, 1999.
[14] K. Maekawa, T. Ishida, T. Kishi. Multi-scale modeling of concrete performance. J Adv Concr Technol 1(2):91-126, 2003. DOI:10.3151/jact.1.91

[15] Y. Zhang, C. Pichler, Y. Yuan, et al. Micromechanics-based multifield framework for early-age concrete. Eng Struct 47:16-24, 2013. DOI:10.1016/j.engstruct.2012.08.015

[16] L. Jendele, V. Šmilauer, J. Červenka. Multiscale hydro-thermo-mechanical model for early-age and mature concrete structures. Adv Eng Softw 72:134-146, 2014. DOI:10.1016/j.advengsoft.2013.05.002.

[17] M. Beneš, I. Pažanin. Homogenization of degenerate coupled transport processes in porous media with memory terms. to appear in Math Meth Appl Sci DOI:10.1002/mma.5718

[18] M. Beneš, R. Štefan. Homogenization of transport processes in early age concrete. AIP Conference Proceedings 2116(1):450028, 2019. DOI:10.1063/1.5114495

[19] J. F. Bourgat. Numerical experiments of the homogenization method. In R. Glowinski, J. L. Lions (eds.), Computing Methods in Applied Sciences and Engineering, 1977, I, pp. 330-356. Springer, 1979. DOI:10.1007/BFb0063630.

[20] R. F. Sviercoski, C. L. Winter, A. W. Warrick. Analytical approximation for the generalized laplace equation with step function coefficient. SIAM J Appl Math 68(5):1268-1281, 2008. DOI:10.1137/070683465

[21] R. F. Sviercoski, B. J. Travis, J. M. Hyman. Analytical effective coefficient and a first-order approximation for linear flow through block permeability inclusions. Comput Math Appl 55(9):2118 2133, 2008. DOI:10.1016/j.camwa.2007.07.016.

[22] R. F. Sviercoski, A. W. Warrick, C. L. Winter. Two-scale analytical homogenization of Richards' equation for flows through block inclusions. Water Resour Res 45(5), 2009. DOI:10.1029/2006WR005598

[23] R. F. Sviercoski, P. Popov, B. J. Travis. Zeroth and first-order homogenized approximations to nonlinear diffusion through block inclusions by an analytical approach. Comput Methods Appl Mech Engrg 198(30):2260-2271, 2009. DOI:10.1016/j.cma.2009.02.020

[24] J. C. Michel, H. Moulinec, P. Suquet. Effective properties of composite materials with periodic microstructure: a computational approach. Comput Methods Appl Mech Engrg 172(1):109-143, 1999. DOI:10.1016/S0045-7825(98)00227-8

[25] Z. Chen, W. Den, H. Ye. Upscaling of a class of nonlinear parabolic equations for the flow transport in heterogeneous porous media. Commun Math Sci 3(4):493-515, 2005. DOI:10.4310/CMS.2005.v3.n4.a2

[26] H. W. Zhang, S. Zhang, J. Y. Bi, B. A. Schrefler. Thermo-mechanical analysis of periodic multiphase materials by a multiscale asymptotic homogenization approach. Int J Numer Meth Eng 69(1):87-113, 2007. DOI:10.1002/nme.1757 
[27] D. Perić, E. A. de Souza Neto, R. A. Feijóo, et al. On micro-to-macro transitions for multi-scale analysis of non-linear heterogeneous materials: unified variational basis and finite element implementation. Int $J$ Numer Meth Eng 87(1-5):149-170, 2011. DOI:10.1002/nme.3014

[28] V. Nguyen, E. Béchet, C. Geuzaine, L. Noels. Imposing periodic boundary condition on arbitrary meshes by polynomial interpolation. Comput Mater Sci $\mathbf{5 5 : 3 9 0 - 4 0 6 , 2 0 1 2 .}$ DOI:10.1016/j.commatsci.2011.10.017.

[29] M. Beneš, I. Pažanin. On degenerate coupled transport processes in porous media with memory phenomena. Z Angew Math Mech 98(6):919-944, 2018. DOI:10.1002/zamm.201700158

[30] P. G. Ciarlet. The Finite Element Method for Elliptic Problems. Elsevier, 1978.

[31] MATLAB. Version 8.6.0 (R2015b). The MathWorks, Inc., Natick, Massachusetts, United States, 2015.
[32] Z. P. Bažant, M. Jirásek. Creep and Hygrothermal Effects in Concrete Structures. Springer, 2018. DOI:10.1007/978-94-024-1138-6.

[33] C. Gonilho Pereira, J. Castro-Gomes, L. Pereira de Oliveira. Influence of natural coarse aggregate size, mineralogy and water content on the permeability of structural concrete. Constr Build Mater 23(2):602-608, 2009. DOI:10.1016/j.conbuildmat.2008.04.009

[34] V. Baroghel-Bouny, M. Mainguy, T. Lassabatere, O. Coussy. Characterization and identification of equilibrium and transfer moisture properties for ordinary and high-performance cementitious materials. Cement Concrete Res 29(8):1225-1238, 1999. DOI:10.1016/S0008-8846(99)00102-7

[35] C. T. Davie, C. J. Pearce, N. Bićanić. Coupled Heat and Moisture Transport in Concrete at Elevated Temperatures-Effects of Capillary Pressure and Adsorbed Water. Numer Heat Tr A-Appl 49(8):733-763, 2006. DOI:10.1080/10407780500503854 